\title{
Ontogeny of midazolam glucuronidation in preterm infants
}

\author{
Saskia N. de Wildt • Greg L. Kearns • Darryl J. Murry • \\ Gideon Koren • John N. van den Anker
}

Received: 10 April 2009 / Accepted: 17 September 2009/Published online: 17 October 2009

(C) The Author(s) 2009. This article is published with open access at Springerlink.com

\begin{abstract}
Purpose In preterm infants, the biotransformation of midazolam (M) to 1-OH-midazolam (OHM) by cytochrome P450 3A4 (CYP3A4) is developmentally immature, but it is currently unknown whether the glucuronidation of OHM to 1-OH-midazolam glucuronide (OHMG) is also decreased. The aim of our study was to investigate the urinary
\end{abstract}

S. N. de Wildt $(\varangle) \cdot$ J. N. van den Anker

Departments of Paediatrics and Paediatric Surgery, Intensive Care, Erasmus MC Sophia Children's Hospital,

Dr Molewaterplein 60, Sk3140,

3015 GJ Rotterdam, The Netherlands

e-mail: s.dewildt@erasmusmc.nl

\section{G. L. Kearns}

Division of Clinical Pharmacology and Medical Toxicology, Children's Mercy Hospitals and Clinics,

Kansas City, MO, USA

\section{G. L. Kearns}

Department of Pediatrics, University of Missouri-Kansas City, Kansas City, MO, USA

\section{J. Murry}

College of Pharmacy, University of Iowa,

Iowa City, USA

\section{G. Koren}

Division of Clinical Pharmacology \& Toxicology,

The Hospital for Sick Children,

Toronto, ON, Canada

\section{G. Koren}

Division of Clinical Pharmacology,

University of Western Ontario,

London, Canada

J. N. van den Anker

Children's National Medical Center, University of Washington,

Washington, D.C., USA excretion of midazolam and its metabolites OHM and OHMG in preterm neonates following the intravenous (IV) or oral (PO) administration of a single $\mathrm{M}$ dose.

Methods Preterm infants (post-natal age 3-13 days, gestational age 26-34 4/7 weeks) scheduled to undergo a stressful procedure received a $30-\mathrm{min}$ IV infusion $(n=15)$ or a PO bolus dose $(n=7)$ of $0.1 \mathrm{mg} / \mathrm{kg}$ midazolam. The percentage of midazolam dose excreted in the urine as $\mathrm{M}$, OHM and OHMG up to $6 \mathrm{~h}$ post-dose was determined.

Results The median percentage of the midazolam dose excreted as $\mathrm{M}, \mathrm{OHM}$ and $\mathrm{OHMG}$ in the urine during the 6-h interval after the IV infusion was $0.44 \%$ (range 0.02 $1.39 \%), 0.04 \%(0.01-0.13 \%)$ and $1.57 \%(0.36-7.7 \%)$, respectively. After administration of the PO bolus dose, the median percentage of $\mathrm{M}, \mathrm{OHM}$ and $\mathrm{OHMG}$ excreted in the urine was $0.11 \%(0.02-0.59 \%), 0.02 \%(0.00-0.10 \%)$ and $1.69 \%(0.58-7.31 \%)$, respectively. The proportion of the IV midazolam dose excreted as OHMG increased significantly with postconceptional age $(r=0.73, p<0.05)$. Conclusion The glucuronidation of OHM appears immature in preterm infants less than 2 weeks of age. The observed increase in urinary excretion of OHMG with postconceptional age likely reflects the combined maturation of glucuronidation and renal function.

Keywords CYP3A · Glucuronidation · Midazolam · Ontogeny $\cdot$ Urinary metabolic ratio

\section{Introduction}

The plasma clearance rate of the benzodiazepine midazolam (M) is widely used as an in vivo surrogate measurement of cytochrome P450 3A4/5 (CYP3A4/5) activity $[1,2]$. We have previously demonstrated that midazolam 
clearance is substantially reduced in preterm infants as compared to older children and adults due to the developmental immaturity of CYP3A4 metabolism (i.e. low CYP3A4 activity) [3]. In adults, the main hydroxylated metabolite of midazolam, 1-OH-midazolam (OHM), is glucuronidated to 1-OH-midazolam glucuronide (OHMG) [4]. In vitro data suggest that UGT2B4, UGT2B7 and, to a lesser extent, UGT1A4, are responsible for the glucuronidation of OHM in humans [5]. Interestingly, it has also been recently shown that midazolam can be directly glucuronidated by UGT1A4, a pathway that becomes more important quantitatively in determining midazolam plasma clearance in individuals with low constitutive CYP3A4/5 activity $[6,7]$.

The UDP-glycosyltransferase genes mainly encode UDP-glucuronosyltransferases (UGTs), a superfamily of enzymes. Four families of genes have been identified within this superfamily: UGT1, UGT2, UGT3 and UGT8. The UGT1 and UGT2 gene families are critical in the detoxification of endogenous and exogenous (e.g. drugs) chemicals, and at least 16 genes have been identified to date in humans [8].

As with CYP3A4, glucuronidation activity shows a developmental surge after birth [9]. However, the exact developmental profiles for the various UGT isoforms have not been elucidated to date. In vitro data suggest that UGT1A4, UGT2B4 and UGT2B7 have a low activity profile immediately after birth that increases to adult levels within the first 1-2 years of life, but the developmental pattern seems to be unique for each individual enzyme [912]. These data have been corroborated by in vivo pharmacokinetic data for morphine [13] and zidovudine [14], both of which are UGT2B7 substrates.

Based on the available data, we hypothesized that the glucuronidation of midazolam and its main metabolite OHM is significantly reduced in preterm infants as compared to adults. While ethical and practical issues limit the number and volume of blood samples that can be obtained to study drug metabolism in preterm infants [15], urinary metabolite profiles can provide an alternative method for examining drug biotransformation [16]. Therefore, we have examined the urinary excretion of midazolam, OHM and OHM glucuronide in preterm neonates after a single midazolam dose with the aim of further elucidating the developmental pattern of 1-OH-midazolam glucuronidation.

\section{Methods}

The samples used for analysis in this current study were obtained from subjects participating in a larger investigation designed to assess the plasma pharmacokinetics and pharmacodynamics of midazolam in preterm neonates $[3$, $17,18]$. The quantification of midazolam and its metabo- lites from urine specimens was not included in the original studies consequent to resource limitations at the time of sample collection.

\section{Patients}

Neonates were eligible for study entry (into the original study) if they were scheduled to undergo a stressful procedure (e.g. tracheal tube suction, elective nasopharyngeal intubation) and had a pre-existing indwelling arterial catheter placed for purposes of medical care. Patients were excluded if they were receiving morphine, dobutamine, dopamine or a drug known to affect CYP3A activity. In addition, patients were excluded from the study if they had significant underlying hemodynamic, renal, hepatic or neurologic dysfunction.

The infants were recruited from the Neonatal Intensive Care Unit of the Erasmus MC-Sophia Children's Hospital. This research protocol was approved by the Human Ethical Committee of the Erasmus MC in Rotterdam. Written, informed consent was obtained from all parents or legal guardians prior to the enrollment of subjects in the study.

\section{Drug administration and sample collection}

Midazolam (Dormicum injection; Roche Laboratories, The Netherlands) was administered intravenously (IV) as a single $0.1 \mathrm{mg} / \mathrm{kg}$ dose in a $5 \%$ glucose solution $(0.03 \mathrm{mg} / \mathrm{ml})$, infused by a syringe pump over a 30 -min period through microbore tubing into a peripheral vein or a central venous catheter. Oral midazolam (PO) at a concentration of $0.5 \mathrm{mg} / \mathrm{ml}$ was diluted with $5 \%$ glucose to a final volume of $0.5 \mathrm{ml}$ and given as a single $0.1 \mathrm{mg} / \mathrm{kg}$ dose through the gastric tube, followed by $0.5 \mathrm{ml}$ of $5 \%$ glucose $5 \%$ to ensure that no midazolam remained in the tube.

Urine samples were collected in 2-h aliquots for up to $6 \mathrm{~h}$ post-dose. The collection method consisted of placing a cotton gauze in the child's diaper that was separated from the diaper absorbing material by a small plastic sheet. Diapers (including gauze and sheet) were weighed before and after each 2-h period to determine urine output. Urine was pressed from the gauze into a collection tube. Incidental fecal material was removed from the diaper as much as possible before weighing. For each urine collection interval, the sample was mixed by repeated inversion, the volume determined and an aliquot $(2-5 \mathrm{ml})$ poured into a screw-capped, polypropylene storage vial which was immediately placed at $-80^{\circ} \mathrm{C}$ until analysis.

As described previously [3], serial blood samples ( $n=8$ at pre-dose and $0.5,1,2,4,6,12$ and $24 \mathrm{~h}$ post-dose) were obtained from an indwelling cannula (placed for clinical management needs not related to the study) after the midazolam dose had been administered. 
Analytical methods

Urine and plasma samples were analyzed for midazolam and OHM by gas chromatography with mass spectrometric detection (Hewlett Packard 6890; Agilent Technologies, Palo Alto, CA) [3]. The urine samples were analyzed before and after $\beta$-glucuronidase incubation, as described earlier [19]. The column used was a J\&W Scientific DB-17 EVDX $(0.2 \mu \mathrm{m} \times 25 \mathrm{~m}$; J\&W Scientific, Folsom, CA). Diazepam (Elkins Sinn, Cherry Hill, NJ), $5 \mu 1$ of a $500 \mathrm{ng} / \mathrm{ml}$ solution, was added to each sample as an internal standard, and solid phase extraction was performed using a Varian Bond Elut column (Varian, Palo Alto, CA). The range of linearity for the analytical method was from 1 to $500 \mathrm{ng} / \mathrm{ml}$ for midazolam, and from 0.5 to $500 \mathrm{ng} / \mathrm{ml}$ for OHM. The inter-day coefficient of variation $(\mathrm{CV})$ was consistently less than $10 \%$ for both midazolam and $\mathrm{OHM}$ at concentrations spanning the range of linearity. The intra-day CVs were also less than $10 \%$ for both midazolam and $\mathrm{OHM}$ at concentrations spanning the range of linearity. The lower limit of quantitation was $1 \mathrm{ng} / \mathrm{ml}$ for midazolam and $0.5 \mathrm{ng} / \mathrm{ml}$ for $\mathrm{OHM}$ using 0.2 - $\mathrm{ml}$ sample volumes. Frozen stability (i.e. $\geq 90 \%$ of original concentration) was documented for midazolam and OHM in urine for a period of 8 years. All samples were analyzed in duplicate, and the resultant mean concentration was used in the pharmacokinetic and statistical analyses.

\section{Pharmacokinetic analysis}

The OHM glucuronide concentration was calculated by subtracting the total OHM concentration from the OHM concentration after de-glucuronidation, which was next corrected for molecular weight (MW; 517.9 and 341.8 for $\mathrm{OHMG}$ and $\mathrm{OHM}$, respectively) using the equation $\mathrm{OHMG}=\mathrm{OHM} \times(517.9 / 341.8)$. Amounts of midazolam and metabolites excreted in the urine were calculated by multiplying the concentration with urine volume per postdose interval. The proportion of dose excreted was calculated by dividing the amount excreted in the urine over all post-dose intervals by the midazolam dose administered, with a correction for molecular weight where applicable (midazolam MW 325.8).
Plasma pharmacokinetic parameters were calculated using non-compartmental pharmacokinetic analysis as described previously [3].

\section{Statistical analysis}

The results are expressed as mean \pm standard deviation (SD) unless stated otherwise. Non-normally distributed data were expressed as median values (range). The association of continuous values (i.e. postnatal age, postconceptional age) and calculated pharmacokinetic parameters are given as the Spearman's (rs) correlation coefficient. All statistical analyses were performed using SPSS software (ver. 13.0; SPSS, Chicago, IL) and assumed a significance limit of $\alpha=0.05$.

\section{Results}

Demographics and data collection

Urine samples suitable for analysis (e.g. complete collections of at least $2 \mathrm{ml}$ per interval) were available from 15 and seven neonates receiving IV and PO midazolam, respectively. Demographic data and clinical characteristics of this study cohort are summarized in Table 1. In one patient after IV dosing, urine could not be collected from 2-4 h and 4-6 h post-doseinterval due to gross fecal contamination of the specimen. Consequently, data from this patient were excluded from the analyses. In two patients receiving IV midazolam, the diaper could not be changed at the $4 \mathrm{~h}$ time point for clinical reasons. Hence, from these patients, urine was collected as a $4 \mathrm{~h}$ aliquot during the $2-6 \mathrm{~h}$ post-dose interval.

Urinary excretion of midazolam and metabolites

The recovery of midazolam and of its metabolites OHM and OHMG showed large inter-individual variation. The total amounts of midazolam, OHM and OHMG [median (range)] recovered in urine up to $6 \mathrm{~h}$ post-IV dose were 396 (21-2080) ng, 5 (11-200) ng and 2253 (561-14681) ng, respectively. Following the PO midazolam dosing, midazolam, OHM and
Table 1 Patient characteristics

NPT, Nasopharyngeal tube; ETT, endotracheal tube; IV, intravenous; PO, oral dose

Values are expressed as median, with the range in parenthesis, unless indicated otherwise

\begin{tabular}{lll}
\hline Patient characteristics & IV midazolam & PO midazolam \\
\hline Total (male/female) $(n)$ & $15(9 / 6)$ & $7(6 / 1)$ \\
Gestational age (weeks) & $276 / 7(262 / 7-344 / 7)$ & $27(26-276 / 7)$ \\
Postnatal age (days) & $7(3-11)$ & $6(5-13)$ \\
Birth weight (grams) & $1040(745-1365)$ & $920(745-1190)$ \\
Study weight (grams) & $980(900-1535)$ & $910(825-1165)$ \\
Ventilation (none/NPT/ETT) & $1 / 6 / 8$ & $0 / 3 / 4$ \\
\hline
\end{tabular}


OHMG were recovered in the urine in amounts of 128 (22562), 21 (2-101) and 2906 (754-9988) ng, respectively.

The percentages of the IV midazolam dose excreted as midazolam, OHM and OHMG in the urine over a 6-h postdose interval were $0.44 \%(0.02-1.39 \%), 0.04 \%(0.01-0.13 \%)$ and $1.57 \%(0.36-7.7 \%)$, respectively. After PO midazolam dosing, the percentage of midazolam, OHM and OHMG was $0.11 \%(0.02-0.59 \%), 0.02 \%(0.00-0.10 \%)$ and $1.69 \%(0.58-$ $7.31 \%$ ), respectively. The percentage of each of the these analytes recovered in the urine was not significantly different after IV or PO midazolam administration.

Midazolam urinary excretion as a surrogate marker for drug metabolism

We found a stronger positive correlation between the proportion of the IV midazolam dose excreted as OHMG and postconceptional age $(r=0.73, p=0.004)$ than between the same proportion and postnatal age $(r=0.47, p=0.09)$, Fig. 1. Similarly, we found a positive correlation between the proportion of PO midazolam excreted as total OHM (glucuronidated and not glucuronidated) and postconceptional age $(r=0.74 p=0.003)$ but not postnatal age $(0.5$, $0.06)$. In contrast, no correlation was apparent between the proportion of midazolam excreted as OHM or midazolam and age. We did not find a postconceptional age-related change in the $\mathrm{M} /$ total OHM urinary ratio (as possible marker of CYP3A activity) after IV midazolam administration $(r=0.303, p=0.29)$. We also did not find a correlation between for bodyweight-corrected midazolam plasma clearance after IV midazolam (also as a surrogate marker of CYP3A activity) and M/total OHM urinary ratio $r=-0.165, p=0.590)$. The addition of the data of seven patients who received the oral midazolam dose (Fig. 1) to the aforementioned analyses of the IV data did not appreciably change the results with respect to significant associations.

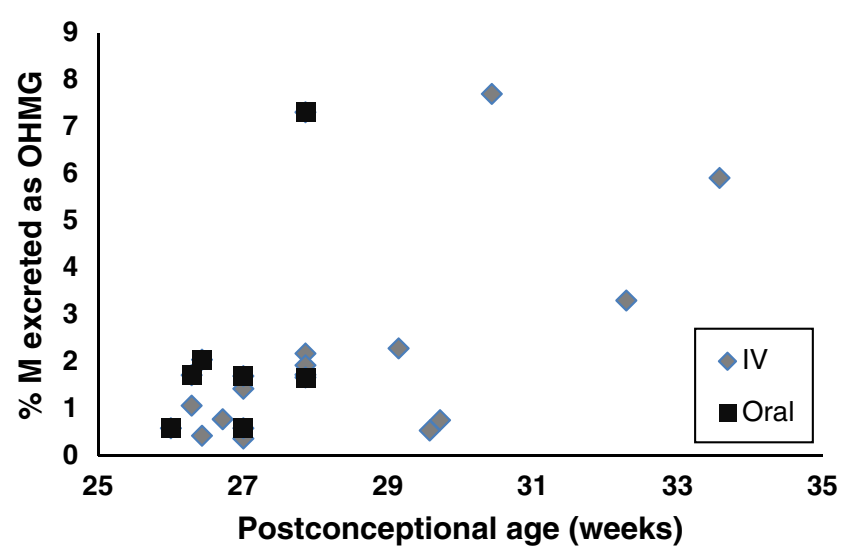

Fig. 1 Effect of postconceptional age on percentage of midazolam $(M)$ excreted in urine as 1-OH-midazolam glucuronide $(O H M G)$

\section{Discussion}

Despite its extensive clinical use in the treatment of neonates and young infants [20], limited data exist which profile the biotransformation and renal excretion of midazolam and its metabolites. The data from this study provide additional insight and understanding of the developmental changes in midazolam disposition in preterm infants.

In the study reported here, the median (range) percentage of midazolam and total OHM (OHM+OHMG) recovered in the urine of preterm infants over a 6 -h period following the IV administration of a single midazolam dose was $0.44 \%$ (range $0.02-1.39 \%$ ) and $1.64 \%(0.37-7.9 \%)$, respectively. This is in contrast to data reported in adults where the median (range) percentage of the midazolam dose recovered in urine up to $6 \mathrm{~h}$ following the administration of a single IV dose was $0.14 \%(0.02-0.40 \%)$ for midazolam and $30.7 \%(1.3-52.6 \%)$ for total OHM [21]. As preterm infants have significantly lower midazolam clearance rates than adults $[3,4]$, the low percentage of midazolam recovered as metabolites in our preterm infants is not unexpected. Considering an average plasma midazolam elimination half-life of 1-2 $\mathrm{h}$ in adults [4], one would predict that $>90 \%$ of a dose would be cleared from the plasma over a 6 $\mathrm{h}$ post-dose time period. In contrast, the midazolam elimination half life in preterm infants $(6-8 \mathrm{~h})$ is significantly longer than that in adults. Hence, during a 6 -h postdose interval in preterm infants, it is possible that only $50 \%$ or less of the total midazolam dose would be cleared from the plasma. This caveat must be considered when our data from preterm infants are compared with those previously reported for adults, [21] especially when using total OHMG excretion as a potential surrogate for the activity of UGT isoforms capable of producing the OHMG metabolite.

In contrast, the percentage of midazolam recovered in the urine as the parent drug appears to be higher in our preterm infants than in adults $[0.44 \%(0.01-1.39 \%)$ vs. $0.14 \%(0.02 \pm 0.40 \%)$, respectively]. This observation may be explained by the higher proportion of urinary midazolam excretion in the presence of developmentally immature CYP3A4/5 activity as an active CYP3A4/5 enzyme which would be expected to markedly reduce midazolam clearance due to drug biotransformation. An analogous situation occurs with caffeine in the neonate where $>50 \%$ of its total clearance in the first few days and weeks of postnatal life results from renal excretion of the unchanged drug, an amount which diminishes over time as the activity of enzymes capable of metabolizing caffeine mature [23, 24].

We have previously shown in the same patient population that the median $\mathrm{OHM} \mathrm{AUC}_{0-\mathrm{t}} / \mathrm{M} \mathrm{AUC}_{0-\mathrm{t}}$ ratio in plasma was low (0.09) with large inter-individual variation (range $<0.001-1$, CV 191\%) when compared to that of older children (2 days to 17 years of age; OHM/M ratio 
$0.14 \pm 0.21)[3,19]$. We speculated that the low OHM/M ratio in the preterm infants was due to developmentally immature CYP3A4/5 activity. However, based on the data reported here, with low (unconjugated) OHM and OHMG urinary excretion, we contend that the low OHM/M plasma ratio observed in preterm infants is more likely to be compounded by immature CYP3A4/5 activity and relatively less immature UGT activity. Unfortunately, due to ethical restraints on blood sample volumes, we were not able to also measure OHMG in plasma, which could have further supported this hypothesis.

We observed a postconceptional age-related increase in urinary excretion of $\mathrm{OHMG}$ and total $\mathrm{OHM}(\mathrm{OHM}+\mathrm{OHMG})$, but not of midazolam or OHM in our patients. This observation also suggests, that independent of a likely increase in OHM formation with age consequent to CYP3A maturation, glucuronidation capacity seems to be more mature than that of CYP3A.

These data should be interpreted with care, however, as in addition to immature drug metabolism, immature renal function in our preterm patient cohort may also have contributed to a lower urinary recovery of midazolam as $\mathrm{OHM}$ and OHMG compared to adults [22]. Urinary metabolite ratios may be dependent on renal function, especially in those instances when the contribution of renal clearance of the parent drug is low in relation to its metabolic clearance [16, 25]. Unfortunately, GFR was not routinely measured in our patients. Due to the relationship between GFR and postconceptional age in preterm infants, in light of the small sample size studies, we were also not able to correct our findings for estimated GFR. However, limited data from a small group of asphyxiated term newborns appear to support our findings in relation to the overall importance of renal function as a determinant of midazolam clearance [26].

Interestingly, recent in vitro data have shown that, in addition to the glucuronidation of the hydroxylated metabolites of midazolam, the parent drug can be metabolized by UGT1A4 to a midazolam N-glucuronide (MG) [6]. In adults, this particular metabolite accounts for only a minor fraction of the midazolam metabolites formed. In the presence of a CYP3A4/5 inhibitor, midazolam is largely biotransformed to midazolam $\mathrm{N}$-glucuronide at the expense of $\mathrm{OHM}(\mathrm{G})$ formation [6]. However, given in vitro data which demonstrate that UGT1A4 activity is reduced during early life, [11], it is likely that developmentally associated reductions in both CYP3A4/5 and UGT activity contribute to overall reductions in midazolam biotransformation in neonates, resulting in renal clearance playing a greater roleproportionally - in drug excretion.

Although we did not find a significant difference in urinary midazolam and metabolite excretion after IV and PO excretion, visual inspection of the data suggest that less midazolam and more OHMG is excreted after oral than after IV administration. This observation could be contributed to first-pass metabolism of orally administered midazolam in the intestine and liver. Possibly, due to the immaturity of intestinal CYP3A, as we showed earlier in our midazolam pharmacokinetic studies, this difference did not reach statistical significance[17]. These results should be interpreted in the context of the small sample size and the large inter-individual variation observed.

As noted above, our study has several limitations that deserve consideration. First, truncation of urine collection to a 6-h post-dose period was necessitated largely by the fact that subjects did not have indwelling urinary catheters and that the application of adhesive-containing external urine collection bags is associated with excoriation risk in neonates. We also observed that the odds of significant fecal contamination of the gauze used for passive urine collection increased as the collection period was extended. Second, as shown for the CYP2D6 probe dextromethorphan, urinary $\mathrm{pH}$ can seriously affect metabolite ratios and, hence, reduce their usefulness as a metabolic probe [27]. Unfortunately, due to our urinary collection method, we were not able to measure urinary $\mathrm{pH}$ directly after the neonate had urinated. Urine was collected over a 2-h period in a gauze, during which time the urinary $\mathrm{pH}$ may have significantly changed. Also, the extent to which the midazolam urinary ratio is affected by urinary $\mathrm{pH}$ has not, to our knowledge, been studied. Finally, our data did not enable true elucidation of the isoform specificity nor did they permit assessment of the potential contribution of renal glucuronidation (by UGT2B4 and UGT2B7) $[28,29]$ to the overall extent of midazolam biotransformation.

In conclusion, our experimental data corroborate previous reports of developmental immaturity in the activity of enzymes known to be responsible for midazolam biotransformation. In sick preterm infants in whom the activity of drug metabolizing enzymes is known to be low, the expected postnatal maturation in renal function provides a quantitatively important route for the clearance of midazolam and its metabolites, with renal clearance predominating during the first few weeks of life. Our data also illustrate the importance of considering the impact of ontogeny on both drug metabolism and renal clearance when examining apparent age-associated differences in drug disposition in the neonatal period.

Acknowledgements Dr. de Wildt was supported by the Sophia Foundation for Scientific Research, the Netherlands while working on this research.

Funding This research was partially supported by an unconditional grant from Roche, USA. Dr. de Wildt received an unconditional grant to partially cover the printing costs for her $\mathrm{PhD}$ thesis and an unconditional traveling grant, both from Roche, the Netherlands. 
Open Access This article is distributed under the terms of the Creative Commons Attribution Noncommercial License which permits any noncommercial use, distribution, and reproduction in any medium, provided the original author(s) and source are credited.

\section{References}

1. Watkins PB (1994) Noninvasive tests of CYP3A enzymes. Pharmacogenetics 4:171-184

2. Streetman DS, Bertino JS Jr, Nafziger AN (2000) Phenotyping of drug-metabolizing enzymes in adults: a review of in-vivo cytochrome P450 phenotyping probes. Pharmacogenetics 10 (3): 187-216

3. de Wildt SN, Kearns GL, Hop WC, Murry DJ, Abdel-Rahman SM, van den Anker JN (2001) Pharmacokinetics and metabolism of intravenous midazolam in preterm infants. Clin Pharmacol Ther 70(6):525-531

4. Heizmann P, Ziegler WH (1981) Excretion and metabolism of 14Cmidazolam in humans following oral dosing. Arzneimittelforschung 31(12a):2220-2223

5. Zhu B, Bush D, Doss GA, Vincent S, Franklin RB, Xu S (2008) Characterization of 1'-hydroxymidazolam glucuronidation in human liver microsomes. Drug Metab Dispos 36(2):331-338

6. Klieber S, Hugla S, Ngo R, Arabeyre-Fabre C, Meunier V, Sadoun F, Fedeli O, Rival M, Bourrie M, Guillou F, Maurel P, Fabre G (2008) Contribution of the N-glucuronidation pathway to the overall in vitro metabolic clearance of midazolam in humans. Drug Metab Dispos 36(5):851-862

7. Hyland R, Osborne T, Payne A, Kempshall S, Logan YR, Ezzeddine K, Jones B (2009) In vitro and in vivo glucuronidation of midazolam in humans. Br J Clin Pharmacol 67(4):445-454

8. Mackenzie PI, Bock KW, Burchell B, Guillemette C, Ikushiro S, Iyanagi T, Miners JO, Owens IS, Nebert DW (2005) Nomenclature update for the mammalian UDP glycosyltransferase (UGT) gene superfamily. Pharmacogenet Genomics 15(10):677-685

9. de Wildt SN, Kearns GL, Leeder JS, van den Anker JN (1999) Glucuronidation in humans. Pharmacogenetic and developmental aspects. Clin Pharmacokinet 36(6):439-452

10. Strassburg CP, Strassburg A, Kneip S, Barut A, Tukey RH, Rodeck B, Manns MP (2002) Developmental aspects of human hepatic drug glucuronidation in young children and adults. Gut 50 (2):259-265

11. Miyagi SJ, Collier AC (2007) Pediatric development of glucuronidation: the ontogeny of hepatic UGT1A4. Drug Metab Dispos 35(9):1587-1592

12. Zaya MJ, Hines RN, Stevens JC (2006) Epirubicin glucuronidation and UGT2B7 developmental expression. Drug Metab Dispos 34(12):2097-2101

13. Bouwmeester NJ, Anderson BJ, Tibboel D, Holford NH (2004) Developmental pharmacokinetics of morphine and its metabolites in neonates, infants and young children. Br J Anaesth 92(2):208217

14. Boucher FD, Modlin JF, Weller S, Ruff A, Mirochnick M, Pelton S, Wilfert C, McKinney R Jr, Crain MJ, Elkins MM et al (1993)
Phase I evaluation of zidovudine administered to infants exposed at birth to the human immunodeficiency virus. J Pediatr 122 (1):137-144

15. Abdel-Rahman SM, Reed MD, Wells TG, Kearns GL (2007) Considerations in the rational design and conduct of phase I/II pediatric clinical trials: avoiding the problems and pitfalls. Clin Pharmacol Ther 81(4):483-494

16. Tucker GT, Rostami-Hodjegan A, Jackson PR (1998) Determination of drug-metabolizing enzyme activity in vivo: pharmacokinetic and statistical issues. Xenobiotica 28(12):1255-1273

17. de Wildt SN, Kearns GL, Hop WC, Murry DJ, Abdel-Rahman SM, van den Anker JN (2002) Pharmacokinetics and metabolism of oral midazolam in preterm infants. Br J Clin Pharmacol 53 (4):390-392

18. de Wildt SN, Kearns GL, Sie SD, Hop WCJ, van den Anker JN (2003) Pharmacodynamics of intravenous and oral midazolam in preterm infants. Clin Drug Invest 23(1):27-38

19. de Wildt SN, de Hoog M, Vinks AA, van der Giessen E, van den Anker JN (2003) Population pharmacokinetics and metabolism of midazolam in pediatric intensive care patients. Crit Care Med 31 (7):1952-1958

20. Blumer JL (1998) Clinical pharmacology of midazolam in infants and children. Clin Pharmacokinet 35(1):37-47

21. Streetman DS, Kashuba AD, Bertino JS Jr, Kulawy R, Rocci ML Jr, Nafziger AN (2001) Use of midazolam urinary metabolic ratios for cytochrome P450 3A (CYP3A) phenotyping. Pharmacogenetics 11 (4):349-355

22. van der Heijden AJ, Grose WF, Ambagtsheer JJ, Provoost AP, Wolff ED, Sauer PJ (1988) Glomerular filtration rate in the preterm infant: the relation to gestational and postnatal age. Eur J Pediatr 148(1):24-28

23. Tserng KY, Takieddine FN, King KC (1983) Developmental aspects of theophylline metabolism in premature infants. Clin Pharmacol Ther 33(4):522-528

24. Thomson AH, Kerr S, Wright S (1996) Population pharmacokinetics of caffeine in neonates and young infants. Therapeutic Drug Monitoring 18(3):245-253

25. Johnson TN, Tucker GT, Rostami-Hodjegan A (2008) Development of CYP2D6 and CYP3A4 in the First Year of Life. Clin Pharmacol Ther 83(5):670-1

26. van Leuven K, Groenendaal F, Toet MC, Schobben AF, Bos SA, de Vries LS, Rademaker CM (2004) Midazolam and amplitudeintegrated EEG in asphyxiated full-term neonates. Acta Paediatr 93(9):1221-1227

27. Ozdemir M, Crewe KH, Tucker GT, Rostami-Hodjegan A (2004) Assessment of in vivo CYP2D6 activity: differential sensitivity of commonly used probes to urine $\mathrm{pH}$. J Clin Pharmacol 44 (12):1398-1404

28. Tukey RH, Strassburg CP (2000) Human UDP-glucuronosyltransferases: metabolism, expression, and disease. Annu Rev Pharmacol Toxicol 40:581-616

29. Gaganis P, Miners JO, Brennan JS, Thomas A, Knights KM (2007) Human renal cortical and medullary UDP-glucuronosyltransferases (UGTs): immunohistochemical localization of UGT2B7 and UGT1A enzymes and kinetic characterization of S-naproxen glucuronidation. J Pharmacol Exp Ther 323(2):422-430 